\title{
Magnetic Resonance Imaging in Neurological Disorders in the Postpartum Period
}

\author{
Chandrashekar H M ${ }^{1}$, Chandana Udayakumar ${ }^{2}$, Naveen J33, Vedaraju K S \\ ${ }^{1}$ Assistant Professor, Department of Radiodiagnosis, Bangalore Medical College and Research Institute, Bangalore, ${ }^{2}$ Senior \\ Resident, Department of Radiodiagnosis, Bangalore Medical College and Research Institute, Bangalore, ${ }^{3}$ Assistant Professor, \\ Department of Radiodiagnosis, Bangalore Medical College and Research Institute, Bangalore, ${ }^{4}$ Professor, Department of \\ Radiodiagnosis, Bangalore Medical College and Research Institute, Bangalore, India
}

Corresponding author: Dr Chandana Udayakumar, Senior Resident Department of Radiodiagnosis, Victoria Hospital, Bangalore Medical College and Research Institute, Bengaluru -02, India

DOI: http://dx.doi.org/10.21276/ijcmsr.2020.5.1.54

How to cite this article: Chandrashekar H M, Chandana Udayakumar, Naveen J, Vedaraju K S. Magnetic resonance imaging in neurological disorders in the postpartum period. International Journal of Contemporary Medicine Surgery and Radiology. 2020;5(1):A247-A250.

\section{A B S T R A C T}

Introduction: Pregnancy and puerperium is a critical period where neurological and hemodynamic disturbances may sometimes lead to serious adverse effects if prompt treatment is not undertaken. The study intended to describe the imaging characteristics of various neurological disorders in the puerperal period.

Material and Methods: Fifty postpartum patients with clinical suspicion of neurological disorders referred to the Department of Radio-Diagnosis from November 2017 to May 2019 underwent MRI of brain. All MR imaging examinations were performed on a Siemens 1.5-T Magnetom Avanto MR system.T1W, T2W axial, coronal and sagittal images, FLAIR, DWI and Gradient sequence images were obtained in all patients. MR angiogram, venogram and contrast enhanced MRI was obtained in specific conditions.

Results: Among the 50 patients, $44 \%$ of the cases were found to be normal and $56 \%$ had findings. Posterior reversible encephalopathy syndrome (22\%) was the most common disorder seen, followed by infarcts (12\%) and cerebral venous thrombosis (10\%). Meningioma, neurocysticercosis, pituitary microadenoma, mesial temporal sclerosis and intraparenchymal hematoma were seen in $2 \%$ of cases each. Clinical features were non-specific and headache was the most common symptom (76\%). Most of the cases of headache were diagnosed as posterior reversible encephalopathy syndrome (72\%).

Conclusion: MRI was found to be a key modality to evaluate various neurological disorders and to arrive at an accurate diagnosis.MRI is a non-invasive imaging modality with no radiation hazard, excellent resolution and multiplanar imaging capability. Use of prompt imaging will be helpful to diagnose and differentiate between various benign and critical neurological illnesses and to start appropriate therapy.

Keywords: Posterior Reversible Encephalopathy Syndrome, Cerebral Venous Thrombosis, Neurological disorders, Magnetic Resonance Imaging

\section{INTRODUCTION}

Physiological changes involving hemodynamic, coagulation and endocrine systems occur during pregnancy and puerperium. ${ }^{1}$ These may result in various neurological disorders like eclamptic encephalopathy, ischemic stroke, cerebral venous thrombosis, intracranial haemorrhage, postpartum cerebral angiopathy and pituitary disorders like pituitary apoplexy, sheehan syndrome and lymphocytic adenohypophysitis. ${ }^{2}$ The neurological disorders can occur either due to normal physiological effects of pregnancy or can be new disorders seen incidentally. ${ }^{3}$ Evaluation of postpartum neurological pathologies is a diagnostic challenge as any abnormal symptom faced in the peri-partum period are considered to be a part of pregnancy and ignored. ${ }^{4}$ Symptoms and signs are non-specific and it can be difficult to diagnose based on clinical grounds alone.
The advent of Magnetic Resonance Imaging (MRI) has proved to be a boon in early and non-invasive diagnosis of pregnancy related neurological complications and also helps in recognition of the characteristic imaging findings of various neurological symptoms..$^{5}$ Even when imaging changes are less specific, knowledge of likely possibilities will lead to a more informed differential diagnosis to be made. ${ }^{2,6}$ Thus, MRI will help to know the common neurological complications affecting our population in post-partum period and help to initiate early appropriate treatment to improve maternal outcome.

The present study is conducted with the objectives of evaluating the neurological disorders that occur during the puerperal period and their characteristic MRI imaging features and to correlate these findings with the clinical picture. 


\section{MATERIAL AND METHODS}

Fifty patients presenting to Department of Radio diagnosis, Bangalore Medical College and Research Institute and its attached hospitals, between November 2017 and May 2019 were subjected to MRI examination of brain after due ethical clearance for the study. Patients presenting within 6 weeks of delivery with recent onset neurological symptoms like headache, seizures, sensory or motor deficit, altered sensorium, blurring of vision or primary lactation failure were included in our study. Patients with pre-existing seizure disorder or epilepsy, claustrophobic patients and other general contraindications to MRI such as pacemakers, aneurysmal clips, cochlear implants and metallic implants or metallic foreign bodies etc were excluded from the study. Based on the history and examination, a clinical diagnosis was made. The procedure was explained to the patients and informed written consent was taken. All MR imaging examinations were performed on a $1.5-\mathrm{T}$ Siemens Avanto Magnetom MR system. T1W, T2W axial, coronal and sagittal images, FLAIR, DWI and Gradient sequence images were obtained in all patients. MR angiogram, venogram and contrast enhanced MRI was obtained in specific conditions.

\section{STATISTICAL ANALYSIS}

Data was analyzed using SPSS 22 version software. Categorical data was represented in the form of Frequencies and proportions. For qualitative data, Chi-square test was used as test of significance. Continuous data was represented as mean and standard deviation.

\section{RESULTS}

Based on the clinical features, most common clinical diagnosis made was intracranial hemorrhage (40\%) followed by cerebral venous thrombosis (36\%) (Graph 1$)$.

On MRI, 44\% ( $n=22)$ of the total cases studied were

\begin{tabular}{|c|c|c|c|c|}
\hline Imaging findings & $\begin{array}{l}\text { Present study } \\
\qquad(n=50)\end{array}$ & $\begin{array}{l}\text { Mugadasweeran et } \mathrm{al}^{7} \\
\qquad(\mathrm{n}=150)\end{array}$ & $\begin{array}{l}\text { Chandrashekaran et al } \\
\qquad(n=120)\end{array}$ & $\begin{array}{l}\text { Kavthale S et al } \\
\qquad(n=40)\end{array}$ \\
\hline PRES & $22 \%(n=11)$ & $21 \%(n=46)$ & $10 \%(n=12)$ & $60 \%(n=24)$ \\
\hline Arterial infarcts & $12 \%(n=6)$ & $8 \%(n=12)$ & $1.6 \%(n=19)$ & $4 \%(n=16)$ \\
\hline CVT & $10 \%(n=5)$ & $15 \%(n=22)$ & $29.1 \%(n=35)$ & $25 \%(n=10)$ \\
\hline
\end{tabular}



a.

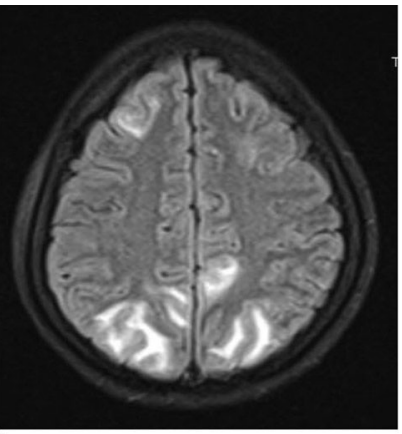

b.

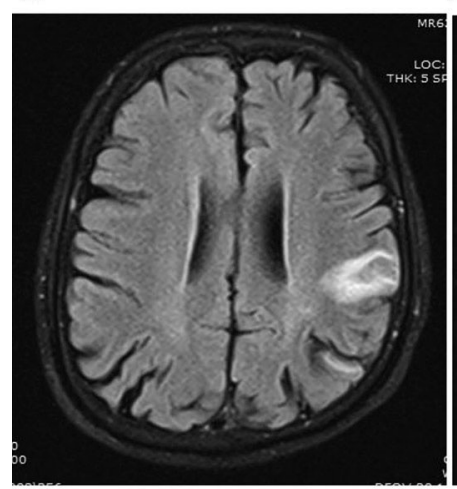

c.

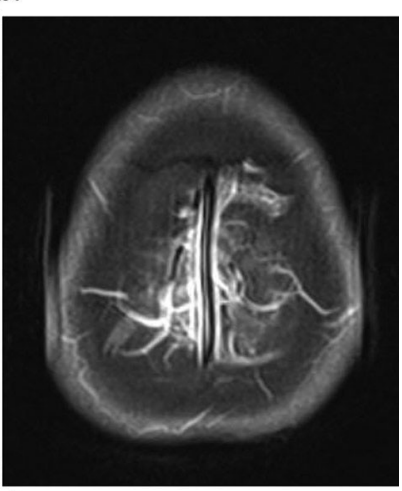

d.

Image-1: PRES in a 28 year old female presenting with history of seizures on post partum day 21, T2 (a) \& FLAIR (b) images showing hyperintense signals in subcortical white matter of bilateral frontal, parietal and occipital lobes. Cerebral venous thrombosis in a 20 year old female on post partum day 11, FLAIR image (c) of brain showing ill defined hyperintense area in cortex and subcortical white matter of left parietal lobe with filling defect in the superior sagittal sinus on $\mathrm{T} 1$ contrast $(\mathrm{d})$.
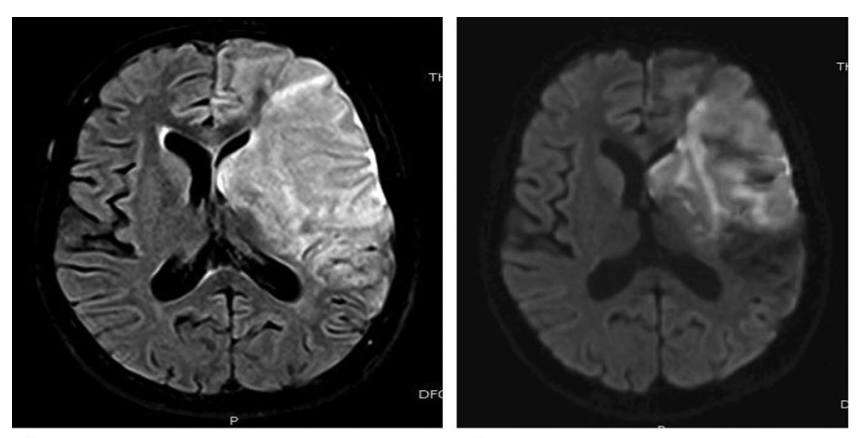

a)

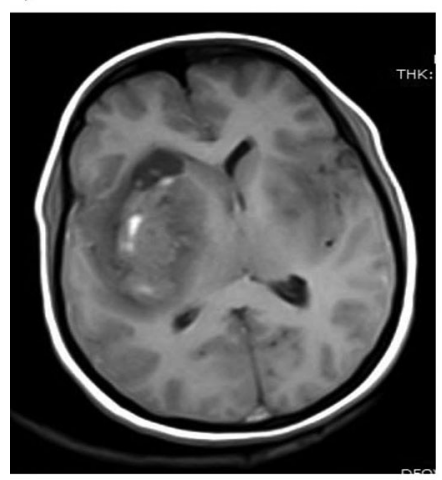

b)

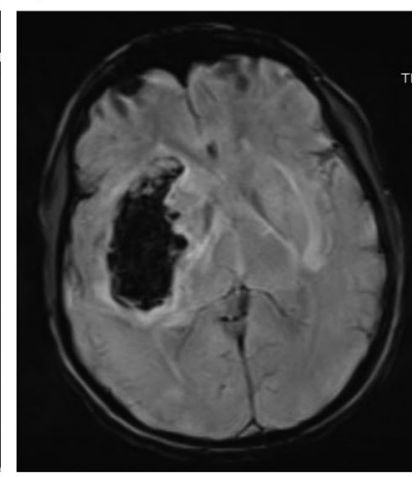

d)

Image-2: Left middle cerebral artery territory infarct in a 29 year old female presenting with history of right hemiparesis on day 2, FLAIR (a) images showing hyperintensity in left fronto temporo parietal lobes, insular cortex \& capsuloganglionic region with corresponding areas of diffusion restriction on DWI (b). Acute intraparenchymal hematoma in a 21 year old female presenting with history of seizures on post partum day 4, showing T1 (c) hyperintense \& GRE (d) blooming suggestive of hematoma. 
found to be normal. Out of the 56\% ( $\mathrm{n}=28)$ of abnormal cases, posterior reversible encephalopathy syndrome was found to be the most common at $22 \%(n=11)$ followed by infarcts which were $12 \%(n=6)$ of the cases. Cerebral venous thrombosis was $10 \%(n=5)$ and tuberculoma, meningioma, neurocysticercosis, pituitary microadenoma, mesial temporal sclerosis and intraparenchymal hematoma were seen in $2 \%$ $(n=1)$ of cases each (Graph 2).

Headache was found to be the most common presenting symptom seen in $76 \%$ of cases $(n=38)$. However, it was found to be a non-specific symptom and also seen in $86.3 \%$ of the normal cases.

Seizure was the second most common symptom for which MRI was done (44\%). It was associated with infarct, intraparenchymal hemorrhage, cerebral venous thrombosis,

\begin{tabular}{|l|c|c|}
\hline Imaging findings & $\begin{array}{c}\text { Present } \\
\text { study } \\
\text { (n=11) }\end{array}$ & $\begin{array}{c}\text { Kavthale } \\
\text { et al } \\
\text { (n=24) }\end{array}$ \\
\hline PRES with infarct & $4 \%$ & $16.6 \%$ \\
\hline PRES with haemorrhage & $4 \%$ & $4.1 \%$ \\
\hline PRES without infarct/haemorrhage & $92 \%$ & $79.1 \%$ \\
\hline \multicolumn{2}{|c|}{$\begin{array}{c}\text { Table-2: Comparison between imaging findings in PRES in our } \\
\text { study and literature }\end{array}$} \\
\hline
\end{tabular}

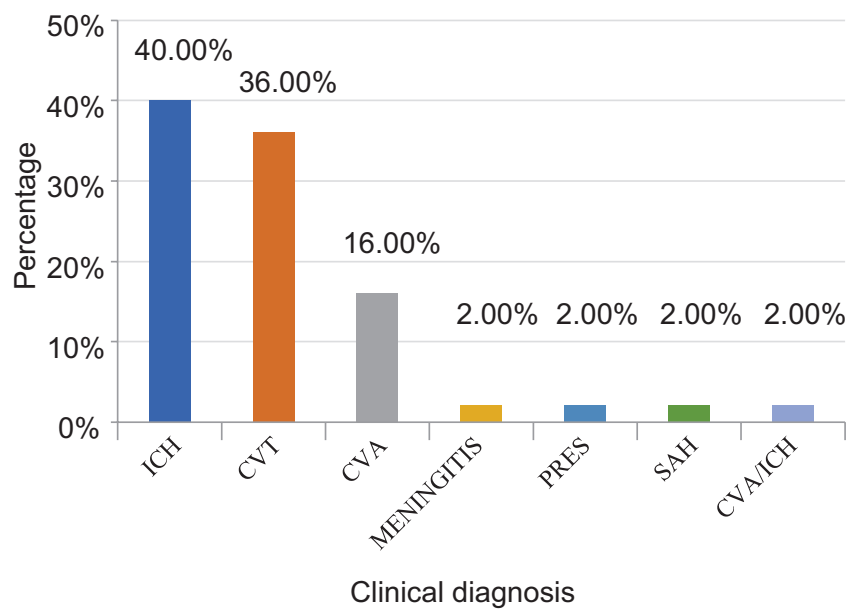

Graph-1: Bar diagram showing distribution of clinical diagnosis in study subjects tuberculoma and posterior reversible encephalopathy. One case of mesial temporal sclerosis was also seen on evaluation for seizures.

Most of the cases diagnosed to have posterior reversible encephalopathy syndrome presented with complaints of seizures and headache (72\%). Headache was presenting symptom in all the cases of cerebral venous thrombosis. In few $(60 \%)$ of the cases, vomiting, seizures and focal neurological deficits was also seen.

\section{DISCUSSION}

The present study was undertaken with an aim of evaluating the neurological disorders occurring during the puerperal period and to correlate magnetic resonance imaging diagnosis of each of these disorders with the clinical picture. Following disorders were noted. (Table 1)

Posterior Reversible Encephalopathy Syndrome (PRES)

Present study showed PRES to be most common finding and represented $22 \%(n=11)$ of the cases. (Image $1-a, b)$ Similar findings were noted in study by Kavthale et $\mathrm{al}^{4}$ where $60 \%$ $(n=24)$ of patients were found to have PRES.

PRES showed involvement of bilateral posterior parietal and occipital lobes involving the cortex and sub cortical white matter (90.9\%). Few cases of atypical PRES were seen with involvement of bilateral midbrain, hemipons and medulla and few cases showed hemorrhagic transformation and infarction. (Table 2)

\section{Cerebral infarction}

Our study showed infarcts in $12 \%(n=6)$ of the cases. Out of these $28.6 \%$ showed haemorrhagic transformation. (Image 2- a,b) A study done by Skidmore et $\mathrm{al}^{7}$ found $58.3 \%$ had ischemic stroke, $30.5 \%$ had haemorrhagic stroke and $11.1 \%$ patients had venous infarcts. One case of intraparenchymal hematoma was also seen in our study. (Image $2-c, d$ )

\section{Cerebral venous thrombosis}

Cerebral venous thrombosis was seen in $10 \%(n=5)$ of the cases in our study. MR venogram showed thrombosis involving superior sagittal sinus (60\% of cases), transverse sinus (20\%) and sigmoid sinus (20\%). (Image 1-c,d) Study by Chandrashekaran et $\mathrm{al}^{8}$ found cerebral venous thrombosis

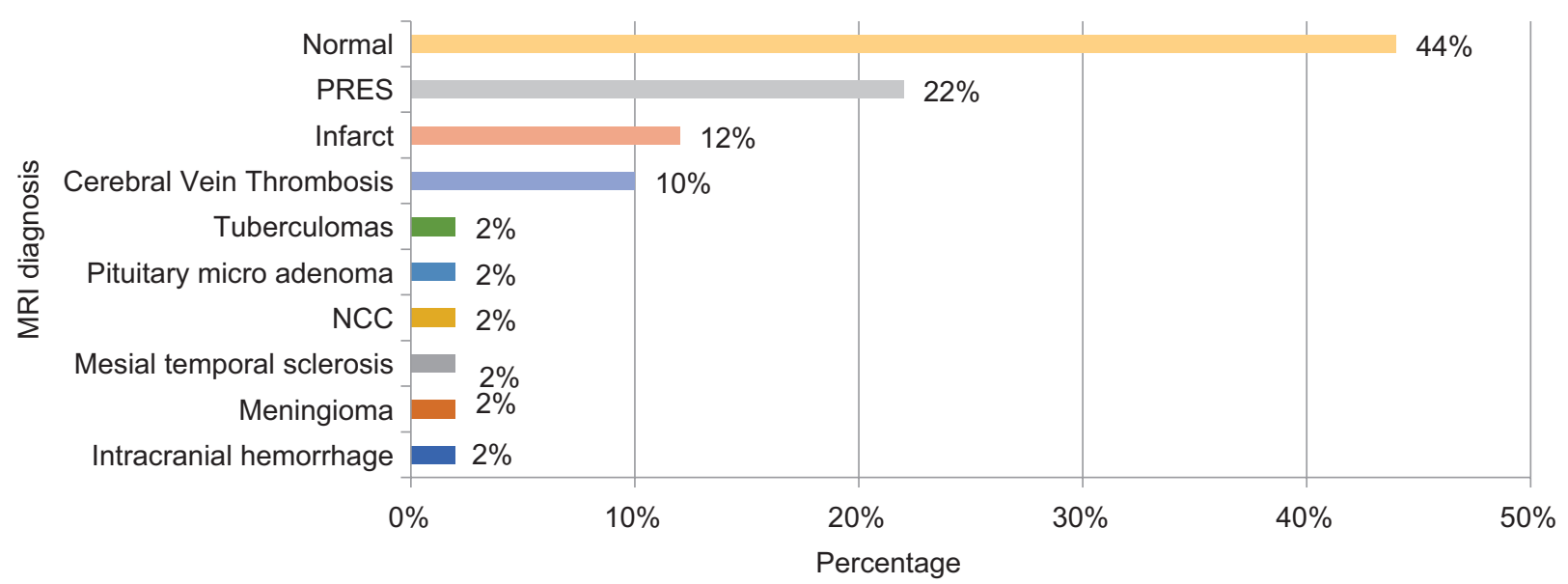

Graph-2: Bar diagram showing MRI diagnosis distribution in study subjects 
to be the most common neurological complication of postpartum period at $29.1 \%(n=35)$ of the cases. Sagittal sinus was involved $45.7 \%$ of the time followed by transverse sinus (25.7\%), sigmoid sinus (17.1\%) and straight sinus (11.4\%). Kavthale et $\mathrm{al}^{4}$ found cerebral venous thrombosis in $25 \%$ $(n=10)$ of the patients in the study, out of which superior sagittal sinus was the commonest to be involved (70\%) followed by the transverse sinus (60\%).

The most common clinical symptom associated with CVT in our study was headache, seen in $100 \%$ of the cases and associated with vomiting and seizures in $60 \%$ of the cases. This was similar to a study conducted by Naveen Thota et $\mathrm{al}^{9}$, where most common symptom was headache (43\%) followed by papilloedema.

\section{Infections}

One case of CNS tuberculosis with multiple tuberculomas was seen in present study which showed multiple ring enhancing lesions in bilateral cerebral hemispheres. Neurocysticercosis was diagnosed in one case which showed multiple ring enhancing lesions with hypointense scolex within.

A study by Gupta $\mathrm{S}$ et $\mathrm{al}^{10}$ showed 12 patients with CNS infections where seven cases (58.3\%) were of tubercular meningitis, four cases (33.3\%) were of acute pyogenic meningitis and one case of viral encephalitis (8.3\%).

\section{Tumors}

One case of meningioma and one case of pituitary microadenoma was seen in our study.

\section{CONCLUSION}

MRI is an excellent modality for detection of pathology and characterization of the neurological disorders in the post partum period. Posterior reversible encephalopathy syndrome was the most common disorder seen, followed by infarcts and cerebral venous thrombosis. MRI was helpful in describing the features and to diagnose various conditions. Most common presenting symptom was headache and most of these patients showed features of posterior reversible encephalopathy syndrome.

Knowledge of the common conditions associated with puerperium is important for early diagnosis and prompt treatment. Good clinical and radiological correlation can help in complete evaluation of these conditions.

\section{REFERENCES}

1. Philips E, Samuels P. Neurological disorders in pregnancy. In: Gabbe SG, Niebyl JR, editors. Gabbe's Textbook of Obstetrics Normal and Problem pregnancies, $7^{\text {th }}$ Ed. Elsevier; 2017: 1030-57

2. Zak TI, Dulai HS, Kish KK. Imaging of Neurologic Disorders Associated with Pregnancy and the Postpartum Period. RadioGraphics. 2007; 27(3):95108.

3. Magudeeswaran PK, Farook AS, Ganesan P, Nataraja V, Rajamani A. A Vital Role of Magnetic Resonance Imaging in Pregnancy-Related Neurological Complications. Int J Sci Stud 2017; 4(12):192-198.

4. Kavthale SS, Kadam MM, Babar M, Shewale V. Study of the imaging features of various neurological pathologies in post-partum period using MRI. MedPulse -
International Medical Journal. 2016; 3(4):418-422.

5. Hacein-Bey L, Varelas PN, Ulmer JL, Mark LP, Raghavan K, Provenzale JM. Imaging of cerebrovascular disease in pregnancy and the puerperium. American Journal of Roentgenology. 2016; 206(1):26-38.

6. Hosley CM, McCullough LD. Acute neurological issues in pregnancy and the peripartum. Neurohospitalist 2011;1 (2):104-116.

7. Skidmore FM, Williams LS, Fradkin KD, Alonso RJ, Biller J. Presentation, etiology, and outcome of stroke in pregnancy and puerperium. Journal of Stroke and Cerebrovascular Diseases. 2001;10(1):1-0.13

8. Chandrasekaran S, Anand PJ, Nanjundan M, Natarajan S, Ramadhas K. Ct and Mr Imaging of Neurological Illnesses in Pregnancy and Puerperium. IOSR Journal of Dental and Medical Sciences 2016;15(4):62-68.

9. Naveen T, Krishna GR, Vengamma B, Dayakar D, Dushyanth J. Clinical manifestations, radiological findings and outcome in cerebral vein and dural sinus thrombosis: a prospective study. Journal of Evidence based Medicine and Healthcare. 2015;2(39):6171-81.

10. Gupta S, Rohatgi Sharma S K, Gurtoo A. A study of neurological disorders during pregnancy and puerperium. Ann Indian Acad Neurol 2006; 9(4):152-7

Source of Support: Nil; Conflict of Interest: None

Submitted: 11-01-2020; Accepted: 19-02-2020; Published online: 22-03-2020 